\title{
Homochirality is originated from handedness of helices
}

\author{
Shubin Liu \\ Research Computing Center, University of North Carolina, Chapel Hill NC 27599-3420 \\ E-mail: shubin@email.unc.edu
}

\begin{abstract}
Homochirality is a common feature of amino acids and carbohydrates, whose origin is still unknown. For example, 19 of 20 natural amino acids are L-chiral but deoxyribose sugars in DNA are always D-chiral. Meanwhile, right-handed helices are ubiquitous in nature. Are these two phenomena intrinsically correlated? Here, we propose that homochirality of amino acids and nucleotide sugars is originated from the handedness of helices. We show that right-handed $3_{10}$-helix and $\alpha$-helix favor the L-chiral form for amino acids, but for deoxyribose sugars right-handed helices prefer the D-chiral form instead. Our analyses unveil that there exist strong cooperativity effects dominated by electrostatic interactions. This work not only resolves the mystery of homochirality by providing a unified explanation for the origin of homochirality in proteins and DNA using helical secondary structures as the root cause, but also ratifies the Principle of Chirality Hierarchy, where chirality of a higher hierarchy dictates that of lower ones. Possible applications of the present work to asymmetric synthesis and macromolecular assembly are discussed.
\end{abstract}

Keywords: Homochirality; Helix; Handedness; Density functional theory; Cooperativity; Electrostatic interaction; Amino acids; DNA; Deoxyribose; Hexahelicene. 
Homochirality is one of the most striking features of life-essential molecules such as proteins and DNA. ${ }^{1-10}$ On the other hand, helix is a shape like a spiral staircase, widely employed by molecules in nature to form stable 3D structures. For instance, in proteins, backbone $\mathrm{N}-\mathrm{H}$ groups can form hydrogen bonds to backbone $\mathrm{C}=\mathrm{O}$ groups located three and four residues earlier along the amino acid sequence, generating $3_{10}$ and $\alpha$ helix secondary structures, respectively. It is well known that helical structures in nature are predominantly righthanded. ${ }^{11-13}$ Handedness is an intrinsic property of the helix, often called axial chirality or one-dimensional (1D) chirality. Chirality arising from chiral centers is called point chirality or zero-dimensional (OD) chirality. In this work, we use the system of $R / S$ absolute configurations to represent chirality. ${ }^{14}$ For amino acids, the chiral $\alpha$-carbon is S-typed except cysteine, which is R-typed, and for deoxyribose monosaccharides in DNA, the 1' carbon linked to a nitrogenous base is always R-typed. ${ }^{15}$

We start with 4 secondary structure models consisting of 6 alanine residues in the form of $\alpha$-helix, $3_{10}$-helix, single-stranded $\beta$-sheet, and double-stranded antiparallel $\beta$-sheet, respectively, shown in Scheme 1. To examine their stability difference between all-R and all-S configurations at the $C_{\alpha}$ position, we build two series of homochiral structures with these 4 models and then consider the total energy difference between these all-R and all-S series using the all-R form as the reference. Table 1 shows the result from a few computational methods and basis sets. It can be seen from the Table that for both single and double stranded $\beta$-sheets, there is no difference in energetics and thus both all-R and all-S enantiomers have the same stability. For the two helices, however, depending on the methodology, a stability difference up to $15 \mathrm{kcal} / \mathrm{mol}$ in favor of the all-S form is possible, 
which qualitatively agrees with what is already known experimentally. Also, comparing the results between $3_{10}$-helix and $\alpha$-helix, we notice that the latter often possesses a larger magnitude of the S-R energy difference, suggesting that $\alpha$-helix prefers more to be in the all$\mathrm{S}$ form than $3_{10}$-helix. Another point from the Table is that the calculated energy difference result considerably depends on the choice of the methodology and basis set.

The second question we address is about non-homochiral helices. Could they be more stable than homochiral enantiomers? To that end, for both $3_{10}$ and $\alpha$ helices consisting of 6 alanine residues, we built a total of $2^{6}=64$ enantiomers with the $C_{\alpha}$ atom of each residue in either R or S form. Table S1 is the result of the energetics analysis, from which we find that all-S enantiomer (the last row) possesses the largest energy difference for both helices, suggesting that homochiral all-S enantiomer is the most stable configuration. From the energy component viewpoint, ${ }^{16-18}$ where the total energy difference $\Delta \mathrm{E}$ comes from three independent contributions, non-interacting kinetic energy $\Delta T_{s}$, exchange- correlation energy $\Delta \mathrm{E}_{\mathrm{xc}}$ and electrostatic energy $\Delta \mathrm{E}_{\mathrm{e}}$,

$$
\Delta \mathrm{E}=\Delta \mathrm{T}_{\mathrm{S}}+\Delta \mathrm{E}_{\mathrm{xc}}+\Delta \mathrm{E}_{\mathrm{e}}
$$

we find that it is the negative value of the electrostatic term $\Delta \mathrm{E}_{\mathrm{e}}$ that is responsible for $\Delta \mathrm{E}<0$, and other two components, $\Delta \mathrm{T}_{\mathrm{S}}$ and $\Delta \mathrm{E}_{\mathrm{xc}}$, are always positive in value and thus they contributed inversely to $\Delta \mathrm{E}$. A dominant contribution from $\Delta \mathrm{E}_{\mathrm{e}}$ suggests that the electrostatic interaction in the all-S form is stronger than that in any other enantiomers. Based on the result from Table 1, the answer to the question is clearly no. Non-homochiral enantiomers are less stable. It is the all-S enantiomer that is most stable.

Does this energy difference depend on the size of helices? The answer is apparently 
yes. To confirm, we build homochiral all-R and all-S enantiomers of $3_{10}$ and $\alpha$ helix models with up to 10 alanine residues and then examine their total energy difference between the two homochiral series. The total energy difference as a function of the number of residues is illustrated in Fig. 1a for $3_{10}$ helix and Fig. $1 \mathrm{~b}$ for $\alpha$ helix. In both cases, the energy difference increases linearly with respect to the number of residues with the correlation coefficient better than 0.99. This result confirms that as the helix grows longer, the magnitude of the total energy difference between all-R and all-S homochiral structures becomes larger. Also, the slope of these two lines is different. For $3_{10}$-helix it is -1.044 , whereas for $\alpha$-helix it is 1.529. For smaller sized helixes, the energy difference between all-S and all-R conformations for $3_{10}$-helix is larger than that for $\alpha$-helix because the former has one more hydrogen bond. However, as the helix size increases, the energy difference for $\alpha$-helix becomes larger. The cross point of the two lines is between 4 and 5 residues.

Earlier, for a noncovalent system consisting of $n$ copies of a repeating unit, we employed the interaction energy per repeating unit of the system, $\Delta \mathrm{E}_{n}$, to categorize cooperativity $\kappa$ with ${ }^{19-21}$

$$
\kappa=-\left(\partial \Delta E_{n} / \partial n\right)
$$

If $\kappa>0$, cooperativity is positive, indicating that an additional unit will induce stronger interactions and thus it makes the entire system more stable. If $\kappa<0$, the consequence is inverse, so cooperativity is negative. If $\kappa=0$, no cooperativity is existent. Here, we extend the above definition to homochiral systems. To that end, $\Delta \mathrm{E}_{n}$ is the total energy difference between all-S and all-R enantiomers $\Delta \mathrm{E}$ divided by the total number of residues $n, \Delta \mathrm{E}_{n} \equiv-|\Delta \mathrm{E}| / n$. The absolute value of $\Delta \mathrm{E}$ is necessary here to make certain that the value of $\Delta \mathrm{E}_{n}$ is always negative, same as 
our previous definition. ${ }^{19-21}$ To visualize the cooperativity behavior of a system, we use the cooperativity profile, where the curve of $\Delta \mathrm{E}_{n}$ as a function of the total number of residues $n$ is plotted. If the curve of the cooperativity profile goes downward, it is positive cooperativity; If it goes upward, it is negative cooperativity.

Figures $1 \mathrm{c}$ and $1 \mathrm{~d}$ illustrates the cooperativity profile of $3_{10}$ helix and $\alpha$ helix, respectively. From these plots, it becomes clear that $\alpha$ helix is positive cooperativity but $3_{10}$ helix is negative cooperativity. This contrasting behavior of cooperativity between these two types of helixes might explain why there are predominantly more $\alpha$ helix motifs in nature than $3_{10}$ helix ones. This cooperativity result is surprising in that if the binding energy is used as the descriptor in Eq. (2) both helices should exhibit positive cooperativity. ${ }^{19}$ However, when we use the energy difference between all-S and all-R homochiral conformations, completely different cooperativity behaviors are obtained for the two kinds of helices.

How about other amino acids besides alanine? Do they behave the same way as alanine? Table 2 shows the energetics result for 8 other amino acids, isoleucine (Ile), leucine (Leu), cysteine (Cys), valine (Val), methionine (Met), serine (Ser), phenylalanine (Phe) and Iysine (Lys), together with alanine (Ala). For each amino acid, we built six-residue homochiral models with the same side chain in both 310 -helix and $\alpha$-helix forms and chirality of six $\mathrm{C}_{\alpha}$ positions took all-R or all-S conformations. From the Table, except Cys, all total energy differences are negative, $\Delta \mathrm{E}<0$, suggesting that the all-S form is energetically more favorable than the all-R form. For Cys, it is well-known that in nature the R-form is favored, so $\Delta \mathrm{E}>0$. Our result agrees well with the experimental evidence. From the energy component viewpoint, as shown by the data in the Table, again, the electrostatic 
contribution plays the dominant role in all cases.

Is it true that right-handed helices always prefer the all-S enantiomer? We had one exception in Table 2 from cysteine, so the answer must be no. Are there other examples? Here, we create derivatives from the right-handed hexahelicene, ${ }^{22}$ where the two outeredged carbon atoms on the three middle benzene rings are each added by one methyl group in either R or S configuration, generating a total of $2^{6}=64$ enantiomers (Scheme 2). Table S2 lists the total energy difference and its three components using, again, the all-R form as the reference. All $\Delta E$ values are positive in the Table, $\Delta E>0$, indicating that for these species, the homochiral R-form is most stable, whereas the all-S form is over $16 \mathrm{kcal} / \mathrm{mol}$ less stable. The result from energy components in the Table suggests that it is again the electrostatic interaction that plays the dominant role because the component $\Delta \mathrm{E}_{\mathrm{e}}$ is most positive, contributing most to make $\Delta \mathrm{E}>0$. These hexahelicene derivatives serve as a solid example showcasing that it is possible that right-handed helices also prefer the all-R enantiomer as the most stable structure.

A more prominent example is deoxyribose monosaccharide in DNA helical structures, where the $1^{\prime}$ carbon linked to a nitrogenous base is always in the all-R form. ${ }^{15}$ Can we apply the idea of the right-handedness of the helical DNA structure to explain homochirality of deoxyribose sugar? Shown in Scheme 3 are two right-handed helical structures as simplified models of the single-stranded DNA, where the phosphate (-O-P $\left.\left(\mathrm{O}_{2}\right)-\mathrm{O}-\right)$ group was replaced by $-\mathrm{O}-\mathrm{C}\left(\mathrm{H}_{2}\right)$-O- linkage, the nucleotide base at the $1^{\prime}$ position of deoxyribose was simplified by either an iso-propyl (iPr) or dimethylamine $\left(\mathrm{NMe}_{2}\right)$ group, and $3^{\prime}$ and $5^{\prime}$ ends were terminated by hydroxyl and methyl groups, respectively. These models are nucleotide 
independent. For each of the two models, we considered up to 10 repeating units, examined two enantiomers with iPr or $\mathrm{NMe}_{2}$ group in either all-R or all-S conformation, and then analyzed the total energy difference and its components using the all- $\mathrm{R}$ form as the reference. To confirm if the all-R homochiral structure is the most stable enantiomer, using the 6-unit structure of Model 1 in Scheme 3 as an example, we built a total of $2^{6}=64$ enantiomers with the $1^{\prime}$ carbon of each deoxyribose unit taking either $\mathrm{R}$ or $\mathrm{S}$ form.

Figure 2 is the result of the two DNA models. Figurers $2 a$ and $2 b$ exhibit the total energy difference $\Delta E$ between all-S and all-R forms as a function of the number of repeating units from 1 to 10 . In both cases, we find that $\Delta E>0$, suggesting that the all-R enantiomer is more stable than the all-S form, in agreement with the experimental evidence. Also, as the number of repeating units increases, so does the total energy difference. The correlation coefficient between $\Delta \mathrm{E}$ and $n$ for the two DNA models equals to 0.990 and 0.983 , respectively. Figures $2 \mathrm{c}$ and $2 \mathrm{~d}$ are the cooperativity profile for the two models. In both cases, the overall trend of the profile goes downward, indicating that they are positively cooperative. Adding additional units into the model systems will make the energy difference more significant, and thus the all-R conformation more stable than the all-S conformation. Since none of these two models includes any hydrogen bonding or other noncovalent interactions, these positive cooperativity effects must come from the handedness of the helix. In real DNA structures where there are strong noncovalent interactions among nucleotide bases, it is expected that the positive cooperativity effect should be even stronger.

Table S3 is the result of 64 enantiomers generated from DNA Model 1 (Scheme 3) to 
demonstrate that non-homochiral structures are less stable than the all-R homochiral structure. As can be seen from the Table, $\Delta E$ is always possible, suggesting that the all- $R$ structure is indeed the most stable enantiomer among these species. From the energy components viewpoint, it is again the stronger electrostatic interaction in the all-R conformation that contributes mostly to $\Delta \mathrm{E}>0$, same as what we have observed in amino acid helices and hexahelicene derivatives.

To have a better understanding about the electrostatic interaction dominance, shown in Fig. 3 are four strong linear correlations of $\Delta \mathrm{E}$ with the component $\Delta \mathrm{E}_{\mathrm{e}}$ from 4 helical systems studied in this work, (a) $3_{10}$ helix, (b) $\alpha$ helix, (c) hexahelicene derivatives, and (d) DNA single-stranded helix Model 1 in Scheme 3. Each model system had 6 chiral centers, and we considered all possible permutations, yielding a total of $2^{6}=64$ conformations. In all these cases, strong linear correlations between $\Delta \mathrm{E}$ and $\Delta \mathrm{E}_{\mathrm{e}}$ are unambiguously seen, with the correlation coefficient equal to $0.919,0.870,0.978$, and 0.931 , respectively. In the cases of $3_{10}$ helix and $\alpha$ helix, it is the strongest electrostatic interaction in the all-S form that makes the all-S enantiomer most stable. In the cases of hexahelicene and DNA deoxyribose models, it is the strongest electrostatic interaction in the all-R form that makes the all- $R$ enantiomer most stable. Even though these models have different homochirality propensity, the dominance of the electrostatic interaction in the most stable structure is always the same.

As another piece of evidence to support the electrostatic interaction dominance, shown in Fig. 4 are the relationships of the total energy difference with the sum of charge differences on oxygen atoms and the $2^{\text {nd }}$-order relative Rényi entropy difference for $3_{10}$-helix 
and $\alpha$-helix. As shown in the plots, there exist strong linear correlations between $\Delta \mathrm{E}$ and these electronic properties. The atomic charge difference on oxygen atoms is of the electrostatic nature. Strong correlations in Figs. 4a and 4c suggest that it is the backbone of the helix that plays the dominant role in determining the value of $\Delta \mathrm{E}$. This result explains why the homochiral propensity does not qualitatively depend on the choice of the side chain, as shown by the results from 9 amino acids in Table 3. The relative Rényi entropy is a relatively new introduction in the literature whose physiochemical significance is still much to be explored. ${ }^{23,24}$ The result in Figs. $4 b$ and $4 d$ implies that this quantity from the information-theoretic approach can be applied to appreciate enantioselectivity.

Thus far, the origin of homochirality in proteins and DNA should have been sufficiently elucidated by the handedness of helices illustrated by our models, which featured the existence of strong cooperativity effect and dominance of the electrostatic interaction. It provides a unified explanation for the origin of homochirality using the secondary structure of proteins and DNA as the root cause. This work also provides the first example about the intrinsic relationship between two kinds of chirality, point (OD) chirality and axial (1D) chirality. Our results in this work show that 1D chirality dictates OD chirality. There are other kinds of chirality in the literature, such as planar (2D) chirality (such as atropisomers), ${ }^{25}$ supramolecular and topological (3D) chirality. ${ }^{26}$ If different kinds of chirality are embedded into one system, as evidenced by the results from this study, the behavior of lower dimension chirality should be governed by the nature of the higher dimension one. We call this the Principle of Chirality Hierarchy. The key factor to make it valid is the existence of strong cooperativity effect dominated by the electrostatic interaction. We anticipate that 
more examples and applications of this principle will be discovered in the future. Even within the same category with multi-level hierarchical structures such as coiled coils, ${ }^{27,28}$ chirality of the higher-level structural hierarchy governs that of the lower level. As important proteinprotein interaction motifs, most coiled coils such as collagen and keratin are left-handed, which is still 1D chirality, but they are built from right-handed $\alpha$-helix coils, whose amino acids are all L-typed.

Finally, with the intrinsic property of helices in favor of the homochiral conformation unveiled in this work, potential applications to asymmetric synthesis and macromolecular assembly are possible. Using axial chirality to design enantioselective products is well-known in the literature ${ }^{29}$ but employing helices as the scaffold to simultaneously generate multiple homochiral centers with the effect of positive cooperativity in place appears to be a new strategy. In this manner, enantioselectivity of products can be more efficiently achieved.

In summary, this work has established the intrinsic relationship between homogeneity of chirality and handedness of helices. We applied this relationship to elucidate the thermodynamic origin of homochirality for amino acids in protein and deoxyribose sugars in DNA. Right-handed $\alpha$-helix and $3_{10}$-helix both favor the L-chiral form of amino acids, whereas deoxyribose sugars in DNA prefer the D-chiral form instead. With the model systems from this study, we have unambiguously validated those propensities. In all these models, the existence of strong cooperativity effect has been identified, which was dominated by the favorable electrostatic interaction in the homochiral conformation. The intrinsic relationship unveiled in this work can be regarded as a special case of the Principle of Chirality Hierarchy, where lower hierarchy chirality is dictated by higher ones. Possible applications of the 
present work to asymmetric synthesis and macromolecular assembly should be possible.

\section{References}

1. Garay, A.D.; Keszthelyi, L.; Demeter, I.; Hrasko, P. Origin of asymmetry in biomolecules. Nature 1974, 250, 332-333.

2. Mason, S.F. Origins of biomolecular handedness. Nature 1984, 311, 19-23.

3. Gidley, D.W.; Rich, A.; Van House, J.; Zitzewitz, P.W. $\beta$ decay and the origin of biological chirality: experimental results. Nature 1985, 297, 639-643.

4. Mason, S.F. Biomolecular homochirality. Chem. Soc. Rev. 1988, 17, $347-359$.

5. Kondepudi, D.K.; Kaufman, R.J.; Singh, N. Chiral symmetry breaking in sodium chlorate crystallization. Science 1990, 250, 975-976.

6. Soai, K.; Shibata, T.; Morioka, H.; Choji K. Asymmetric autocatalysis and amplification of enatiomeric excess of a chiral molecule. Nature 1995, 378, 767-768.

7. Klussmann, M.; Iwamura, H.; Mathew, S. P.; Wells, D.H. Jr.; Pandya, U.; Armstrong, A.; Blackmond. D. G. Thermodynamic control of asymmetric amplification in amino acid catalysis. Nature 2006, 441, 621-623.

8. Hein, J.E.; Blackmond, D.G. On the origin of single chirality of amino acids and sugars in biogenesis. Acc. Chem. Res. 2012, 45, 2045-2054.

9. Skolnick, J.; Zhou, H.; Gao, M. On the possible origin of protein homochirality, structure, and biochemical function, Proc. Natl. Acad. Sci. 2019, 116, 26571-26579.

10. Blackmond, D.G. Autocatalytic models for the origin of biological homochirality. Chem. Rev. 2020, DOI: 10.1021/acs.chemrev.9b00557.

11. Wang, A.J.; Quigley, G.J.; Kolpak, F.J.; van der Marel, G.; van Boom, J.H.; Rich, A. Lefthanded double helical DNA: variations in the backbone conformation, Science 1981, 211, $171-176$.

12. Blaber, M.; Zhang, X.J.; Matthews, B.W. Structural basis of amino acid alpha helix propensity, Science 1993, 260, $1637-1640$

13. Novotby, M.; Kleywegt, G.J. A survey of left-handed helices in protein structures, J. Mol. Biol. 2005, 347, $231-241$. 
14. McMurry, J.; Simanek, E. Fundamentals of Organic Chemistry (Brooks Cole, ed. 6, 2006).

15. Nelson, D.L.; Cox, M.M. Principles of Biochemistry (W. H. Freeman, New York, ed. 4, 2005).

16. Parr, R.G.; Yang, W.T. Density Functional Theory of Atoms and Molecules (Oxford University, New York, 1989).

17. Liu, S.B. Steric effect: A quantitative description from density functional theory. J. Chem. Phys. 2007, 126, 244103.

18. Liu, S.B. Origin and nature of bond rotation barriers: A unified view, J. Phys. Chem. A 2013, 17, 962-965.

19. Rong, C.; Zhao, D.; Yu, D.; Liu, S.B. Quantification and origin of cooperativity: insights from density functional reactivity theory, Phys. Chem. Chem. Phys. 2018, 20, 17990-17998.

20. Zhou, T.; Liu, S.; Yu, D.; Zhao, D.; Rong, C.; Liu, S.B. On the negative cooperativity of argon clusters containing one lithium cation or fluorine anion, Chem. Phys. Lett. 2019, 716, 192 198.

21. Rong, C.; Zhao, D.; Zhou, T.; Liu, S.; Yu, D.; Liu, S.B. Homogeneous molecular systems are positively cooperative but charged molecular systems are negatively cooperative, J. Phys. Chem. Lett. 2019, 10, $1716-1721$.

22. Shen, Y.; Chen, C.F. Helicenes: Synthesis and Applications, Chem. Rev. 2012, 112, 1463-1535.

23. Liu, S.B. Information-theoretic approach in density functional reactivity theory. Acta Phys.-Chim. Sin. 2016, 32, 98-118.

24. Rong, C.; Wang, B.; Zhao, D.; Liu, S.B. Information-theoretic approach in density functional theory and its recent applications to chemical problems. WIREs Comput. Mol. Sci. 2019, 2019, e1461.

25. Elemans, J.A.A.W.; Cat, I.D.; Xu, H.; Feyter, S.D. Two-dimensional chirality at liquid-solid interfaces, Chem. Soc. Rev. 2009, 38, 722-736.

26. Randić, M. Graph theoretical descriptors of two-dimensional chirality with possible extension to three-dimensional chirality, J. Chem. Inf. Comput. Sci. 2001, 41, 639-649.

27. Lupas, A. Coiled coils: new structures and new functions, Trends Biochem. Sci. 1996, 21, $375-382$.

28. Apostolovic, B.; Danial, M.; Al Klok, H. Coiled coils: attractive protein folding motifs for 
the fabrication of self-assembled, responsive and bioactive materials, Chem Soc. Rev. 2010, 39, 3541-3575.

29. Ma, C.; Jiang, F.; Sheng, F.T.; Jiao, Y.; Mei, G.J.; Shi, F. Design and catalytic asymmetric construction of axially chiral 3,3'-bisindole skeletons, Angew. Chem. Int. Ed. 2019, 131, 30463052. 
Table 1. Benchmark results of the total energy difference between all-R and all-S configurations using the all-R form as the reference (S-R) for 4 secondary structure models consisting of 6 alanine residues (Scheme 1 ) with 13 basis sets and density functionals. ${ }^{1}$ Units in $\mathrm{kcal} / \mathrm{mol}$.

\begin{tabular}{|c|c|c|c|c|c|c|c|c|c|}
\hline Basis Sets & $3_{10}-$ Helix & $\alpha$-Helix & $\begin{array}{r}\text { SS } \\
\beta \text {-Sheet }\end{array}$ & $\begin{array}{r}\text { Antiparallel } \\
\beta \text {-Sheet }\end{array}$ & Methods & $3_{10}-$ Helix & $\alpha$-Helix & $\begin{array}{r}\text { SS } \\
\beta \text {-Sheet }\end{array}$ & $\begin{array}{r}\text { Antiparallel } \\
\beta \text {-Sheet }\end{array}$ \\
\hline $6-31 G(d)$ & -6.86 & -6.88 & 0.00 & 0.00 & BLYP & -9.01 & -12.24 & 0.01 & 0.00 \\
\hline $6-31+G(d)$ & -9.89 & -12.34 & 0.00 & 0.00 & B3LYP & -8.69 & -11.37 & 0.01 & 0.00 \\
\hline $6-311 G(d)$ & -7.11 & -8.24 & 0.00 & 0.00 & O3LYP & -12.43 & -15.30 & 0.00 & 0.00 \\
\hline $6-311 G(d, p)$ & -6.41 & -7.83 & 0.00 & 0.00 & PW91 & -7.83 & -10.29 & 0.00 & -0.01 \\
\hline $6-311+G(d)$ & -9.12 & -11.64 & 0.00 & 0.00 & CAM-B3LYP & -7.85 & -9.44 & 0.00 & 0.00 \\
\hline $6-311+G(d, p)$ & -8.69 & -11.37 & 0.00 & 0.00 & B3PW91 & -8.13 & -11.62 & 0.00 & -0.01 \\
\hline $6-311++G(d, p)$ & -8.68 & -11.29 & 0.00 & -0.01 & PBEPBE & -8.07 & -11.10 & 0.00 & -0.01 \\
\hline Def2SVPP & -5.33 & -5.10 & 0.00 & 0.00 & HSEH1PBE & -7.60 & -9.81 & 0.00 & 0.00 \\
\hline Def2TZVP & -9.36 & -11.63 & 0.00 & 0.00 & $\mathrm{HCTH}$ & -13.46 & -14.48 & 0.00 & 0.01 \\
\hline DGDZVP & -9.55 & -11.40 & 0.00 & 0.00 & TPSS & -7.06 & -10.57 & 0.00 & 0.00 \\
\hline cc-pVDZ & -5.02 & -5.13 & 0.00 & 0.00 & wB97XD & -7.29 & -8.12 & 0.00 & 0.00 \\
\hline cc-pVTZ & -8.70 & -10.78 & 0.00 & 0.00 & M06-2X & -5.78 & -4.99 & 0.00 & -0.01 \\
\hline CBSB7 & -6.41 & -7.83 & 0.00 & 0.00 & $\mathrm{RHF}$ & -10.32 & -12.04 & 0.00 & 0.00 \\
\hline
\end{tabular}

${ }^{1}$ In the basis set test, B3LYP functional was employed; in the methodology benchmark, 6-311+G(d,p) basis set was utilized. 
Table 2. The total energy difference and its three components between 6-residue all-R and all-S enantiomers of 9 amino acids in either $3_{10}$ or $\alpha$ helix structure using the all-R form as the reference (S-R). ${ }^{1}$ Units in $\mathrm{kcal} / \mathrm{mol}$.

\begin{tabular}{|c|c|c|c|c|c|c|c|c|}
\hline \multirow{2}{*}{$\begin{array}{l}\text { Amino } \\
\text { Acid }\end{array}$} & \multicolumn{4}{|c|}{$3_{10}-$ Helix } & \multicolumn{4}{|c|}{$\alpha$-Helix } \\
\hline & $\Delta \mathrm{E}$ & $\Delta T_{s}$ & $\Delta \mathrm{E}_{\mathrm{xc}}$ & $\Delta \mathrm{E}_{\mathrm{e}}$ & $\Delta \mathrm{E}$ & $\Delta T_{s}$ & $\Delta \mathrm{E}_{\mathrm{xc}}$ & $\Delta \mathrm{E}_{\mathrm{e}}$ \\
\hline Ala & -7.84 & 21.19 & 4.30 & -33.32 & -8.38 & 31.78 & 2.33 & -42.49 \\
\hline lle & -8.11 & 39.19 & 8.59 & -55.95 & -11.98 & 32.93 & 6.72 & -51.60 \\
\hline Leu & -16.60 & 17.91 & 2.99 & -37.50 & -13.18 & 40.39 & -2.04 & -51.53 \\
\hline Cys & 1.93 & -19.77 & -6.64 & 28.33 & 2.08 & -28.36 & -3.44 & 33.86 \\
\hline Val & -10.25 & 32.29 & 2.93 & -45.47 & -11.15 & 27.30 & 0.60 & -39.05 \\
\hline Met & -9.08 & 14.45 & 0.61 & -24.12 & -13.12 & 24.06 & 0.93 & -38.13 \\
\hline Ser & -7.61 & 32.56 & -1.82 & -38.33 & -1.65 & 25.42 & 4.38 & -31.43 \\
\hline Phe & -1.56 & 14.44 & 1.75 & -17.73 & -3.80 & 9.47 & -0.71 & -12.50 \\
\hline Lys & -1.29 & -0.21 & 8.66 & -9.77 & -12.12 & 31.24 & -0.28 & -43.06 \\
\hline
\end{tabular}

${ }^{1}$ The $\mathrm{N}$-terminal and C-terminal groups of these models are acetyl (ACE) and N-Me amide (NME), respectively; All structures were optimized using the wB97XD density functional and 6-31G(d) basis set for $\mathrm{C}$ and $\mathrm{H}$ atoms and $6-311+\mathrm{G}(\mathrm{d})$ basis set for $\mathrm{N}$ and $\mathrm{O}$ atoms. 


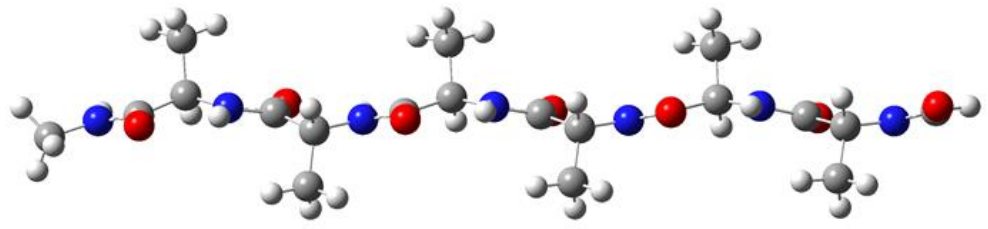

(a) Single-Stranded $\beta$-sheet

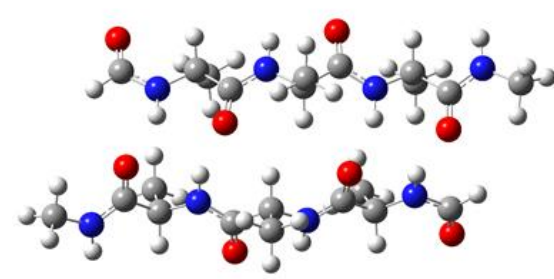

(b) Double-Stranded Antiparallel $\beta$-sheet

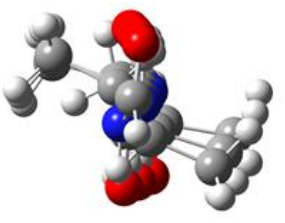

Top View

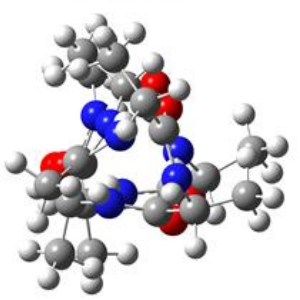

(c) $3_{10}$ helix - Top View

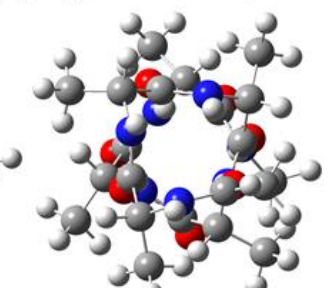

(d) $\alpha$ Helix - Top View

(c) $3_{10}$ Helix - Side View

(d) $\alpha$ Helix -Side View

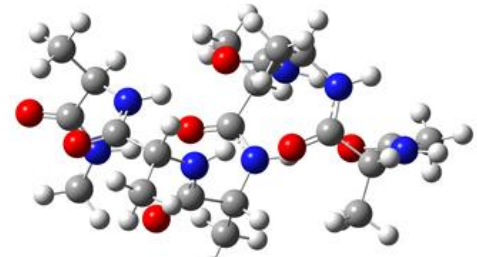

Scheme 1. Four secondary-structure models studied in this work: (a) single-stranded $\beta$-sheet; (b) double-stranded $\beta$-sheet; (c) $3_{10}$-helix; and (d) $\alpha$-helix. Illustrated above are models with six residues, but in this study we considered models up to 10 residues. Both $R$ and $S$ conformations of the $C_{\alpha}$ atom were investigated. For the two 6-residue right-handed helix models, (c) and (d), a total of $2^{6}=64$ enantiomers were examined to study their stability propensities. 


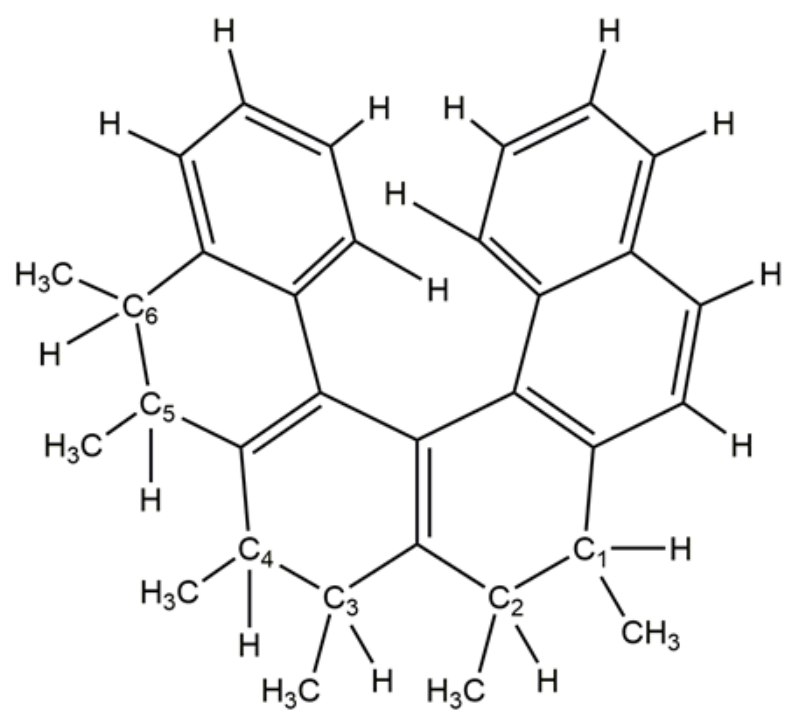

Hexahelicene Derivative Model

Scheme 2. The hexahelicene derivative model in the form of the right-handed helical structure studied in this work. Both $\mathrm{R}$ and $\mathrm{S}$ conformations of the six chiral center, $\mathrm{C}_{1}-\mathrm{C}_{6}$, were investigated, leading to a total of $2^{6}=64$ enantiomers. 


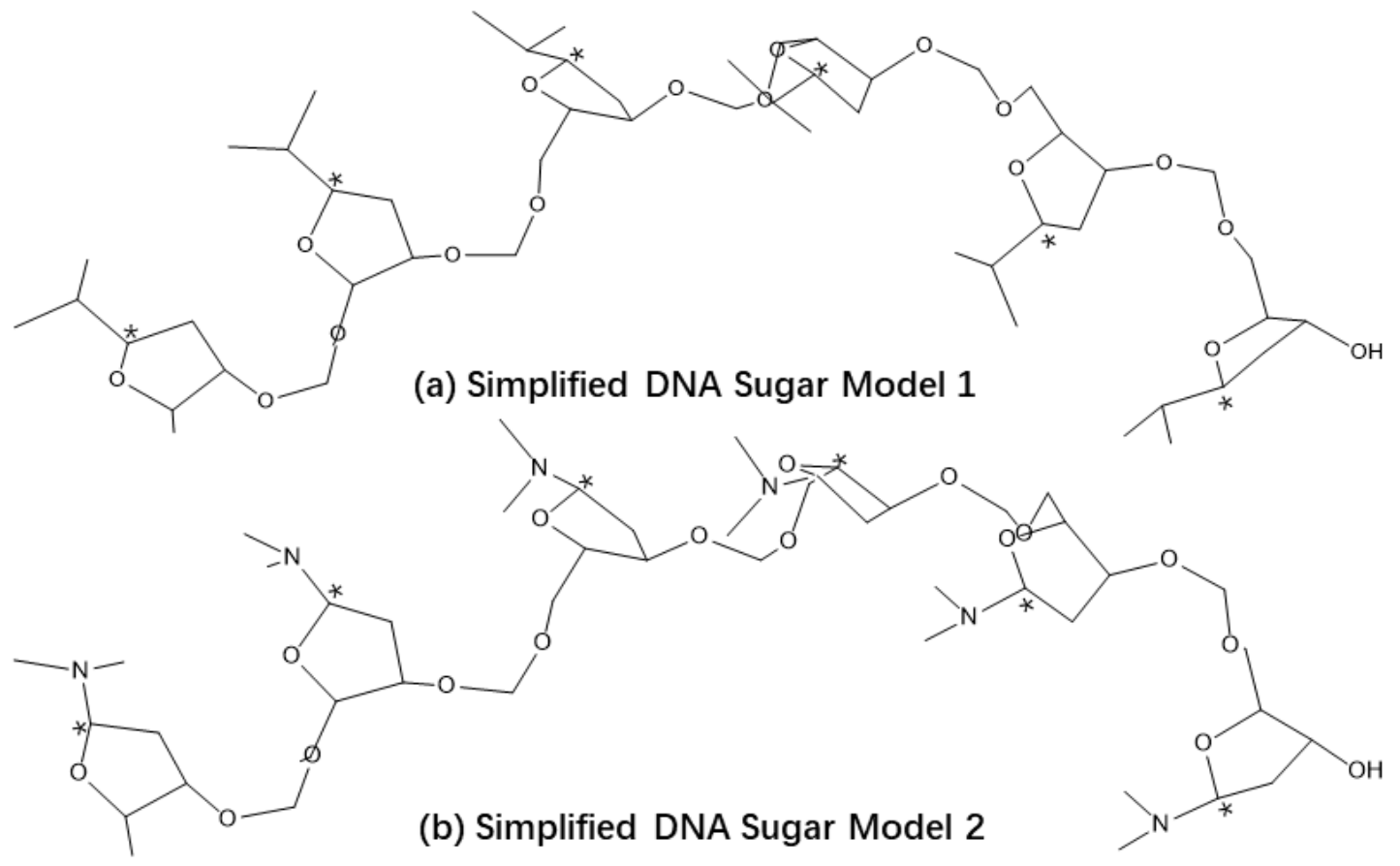

Scheme 3. Two simplified single-stranded DNA deoxyribose models in the form of the righthanded helical structure. In these models, phosphate $\left(-\mathrm{PO}_{4}\right)$ was replaced by $-\mathrm{O}-\mathrm{CH}_{2}-\mathrm{O}-$ linkage and the nucleotide base at the $1^{\prime}$ position of deoxyribose marked by asterisk (*) symbols was substituted by (a) the iso-propyl (iPr) group in Model 1 or (b) dimethylamine $\left(\mathrm{NMe}_{2}\right)$ group in Model 2. The $5^{\prime}$ end of the models is capped by a methyl group and the $3^{\prime}$ end is terminated by a hydroxyl group. We considered two scenarios for each model with $\mathrm{iPr}$ or $\mathrm{NMe}_{2}$ group at either $\mathrm{R}$ or $\mathrm{S}$ conformation. Th other two chiral centers of the deoxyribose sugar are in the same conformation as the regular DNA structure. Illustrated above are models with six repeating units only, but in this study we examined models up to 10 repeating units. 

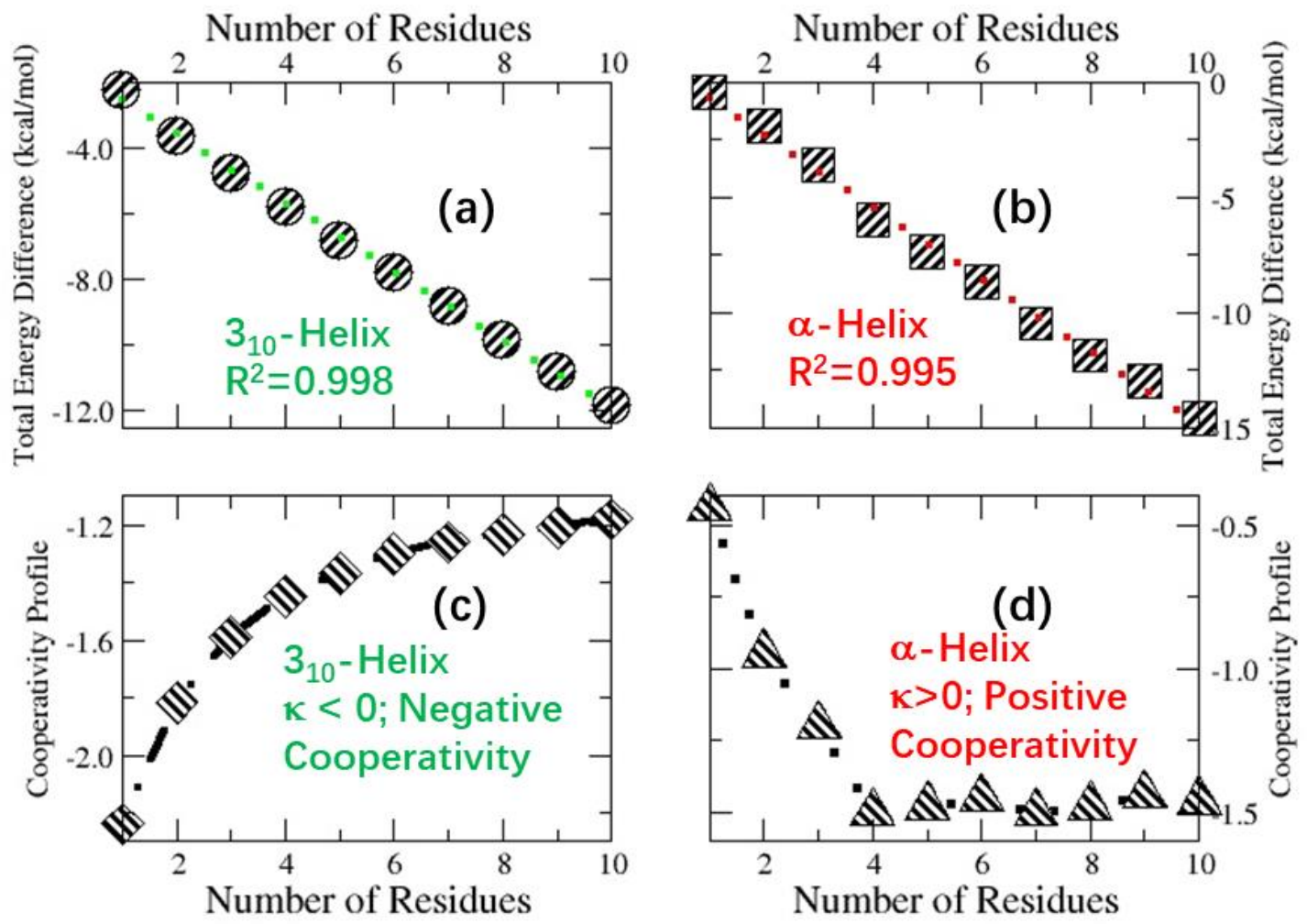

Figure 1. (a) The total energy difference $\Delta E$ between all-R and all-S enantiomers of the righthanded (a) $3_{10}$-helix and (b) $\alpha$-helix as a function of the number of alanine residues from 1 to 10 , and the cooperativity profile of (c) $3_{10}$-helix and (d) $\alpha$-helix up to 10 residues. The corresponding all-R enantiomer of each model system was employed as the reference. 

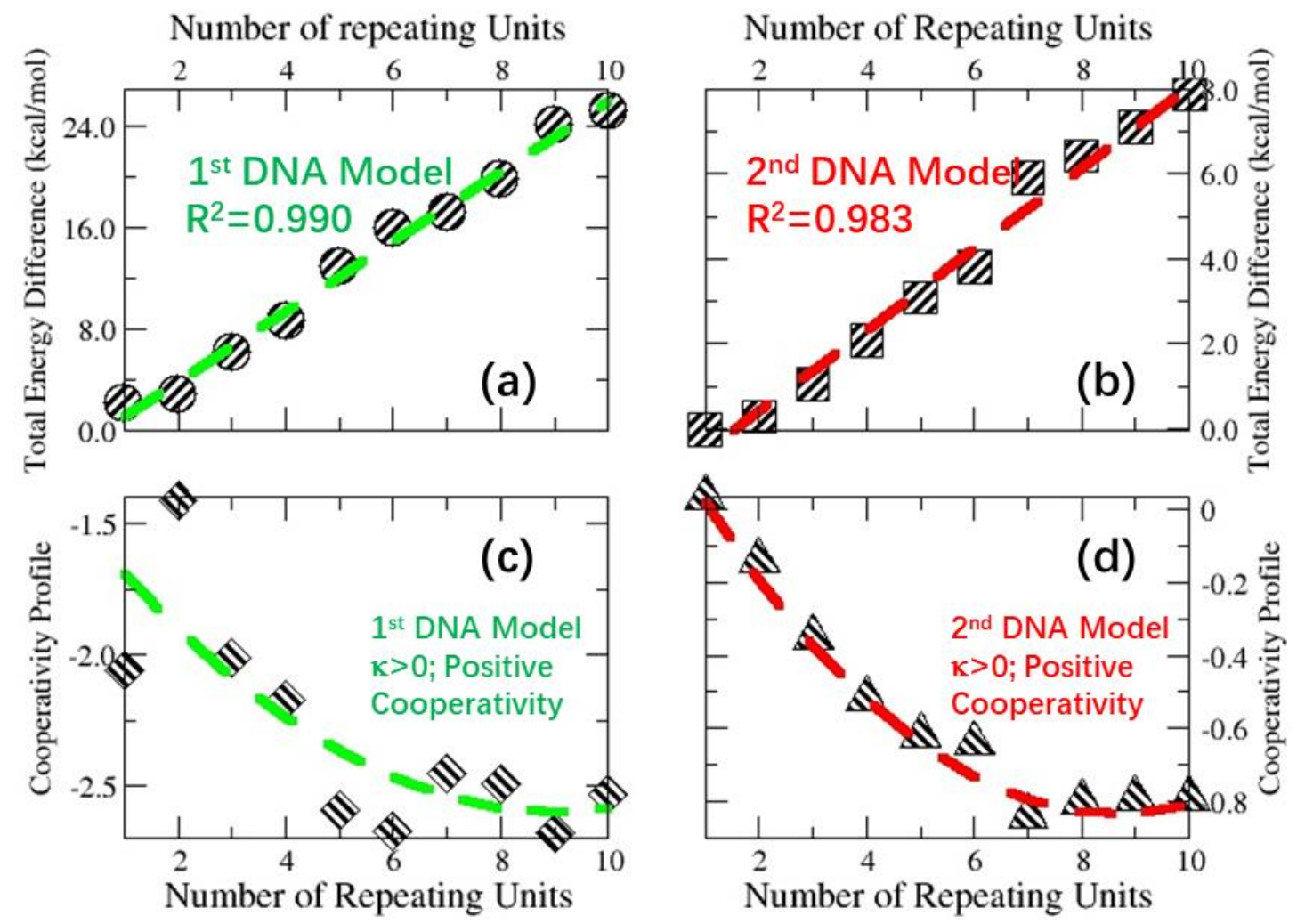

Figure 2. (a) The total energy difference $\Delta \mathrm{E}$ between all-R and all-S enantiomers of the righthanded helix models for the two single-stranded DNA models with the nucleotide base replaced by: (a) iso-propane and (b) dimethylamine, as a function of the number of repeating units from 1 to 10 , and (c) and (d) the cooperativity profiles of the two corresponding models. The corresponding all-R enantiomer of each model system was employed as the reference. 


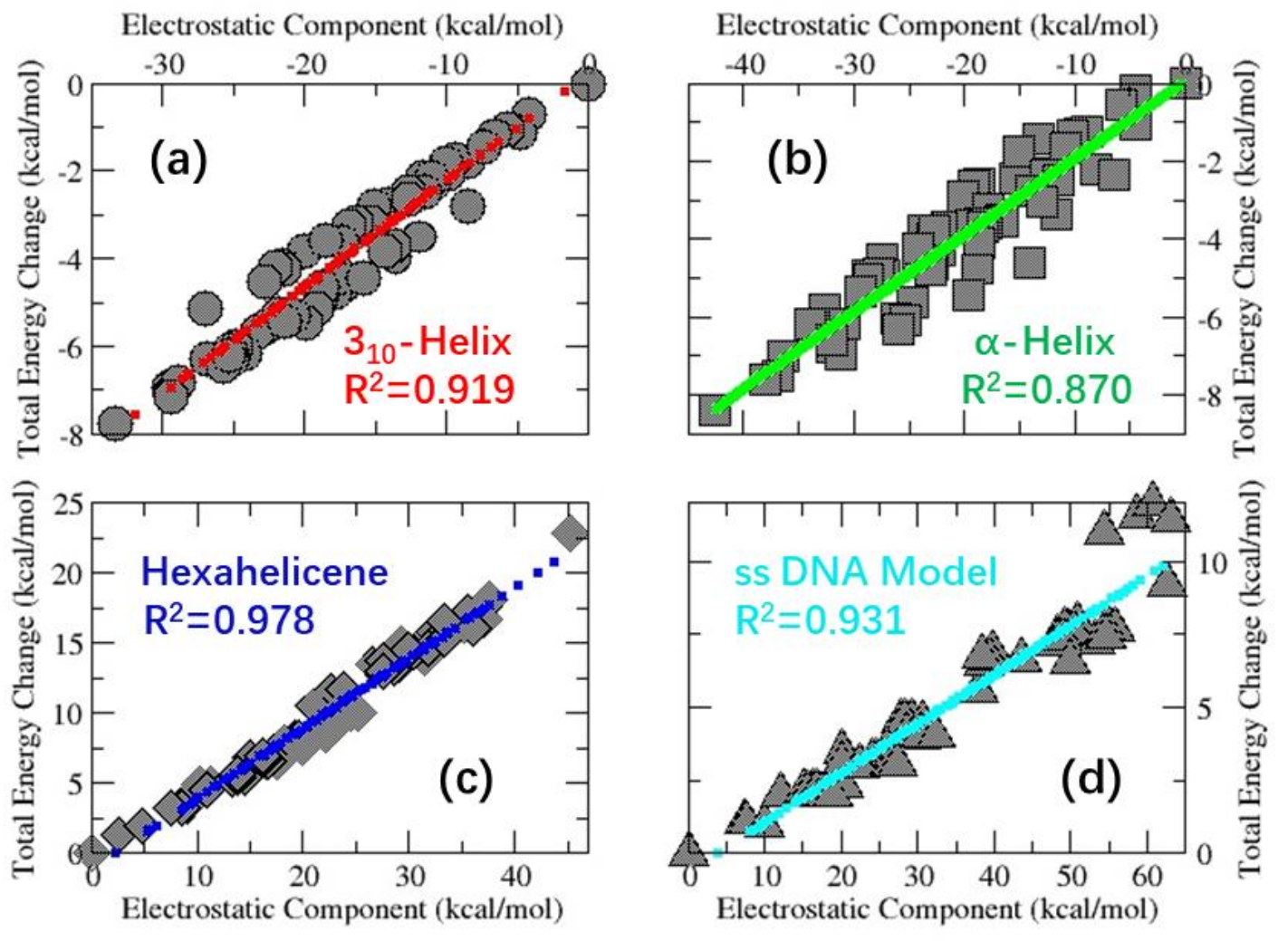

Figure 3. Strong linear relationships between the total energy difference $\Delta E$ and the electrostatic component $\Delta E_{\mathrm{e}}$ for four model systems studied in this work: (a) $3_{10}$-helix; (b) $\alpha$ helix; (c) hexahelicene derivative; and (d) simplified single-stranded DNA Model 1 in Scheme 3 with the nucleotide base replaced by the isopropyl group. In each system, there are 6 chiral centers, each of which is in either $R$ or $S$ conformation, yielding a total of $2^{6}=64$ enantiomers. The reference is the corresponding all-R enantiomer. 


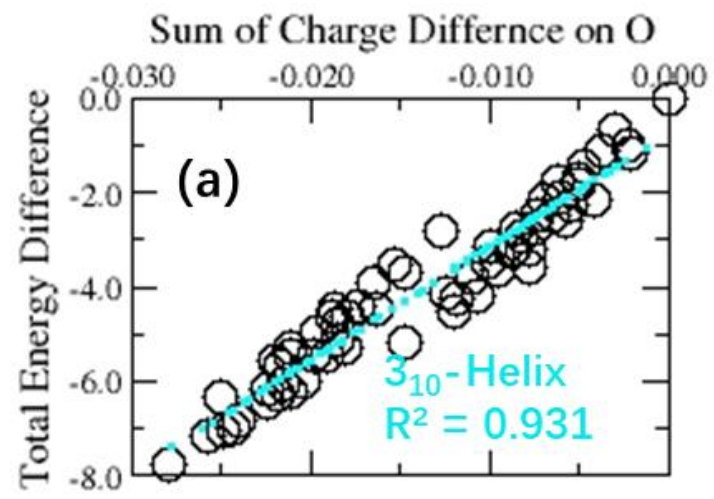

Relative Renyi Entropy Difference
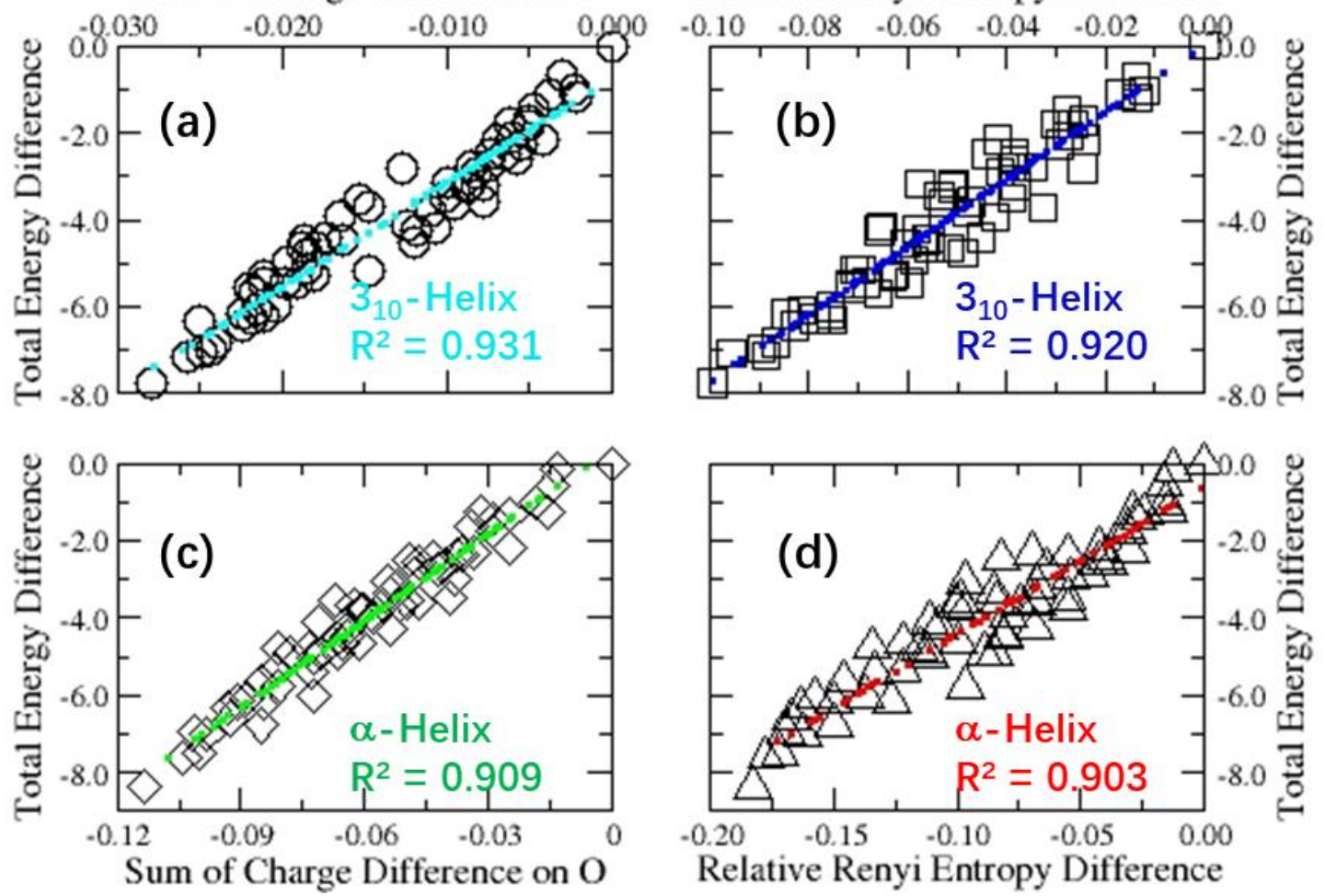

Figure 4. Strong linear relationships of the total energy difference with the sum of charge differences on oxygen atoms and $2^{\text {nd }}$-order relative Rényi entropy difference for 64 enantiomers of both ( $a$ and $b$ ) $3_{10}$ helix and ( $c$ and d) $\alpha$ helices with the corresponding all-R homochiral enantiomer as the reference 